\title{
Estratégias educativas para pessoas com estomia intestinal: revisão integrativa
}

\author{
Educational strategies for people with ostomy bowel: \\ integrative review
}

\author{
Adriana Rodrigues Alves de Sousa ${ }^{1} \bullet$ Luciana Catunda Gomes de Menezes $^{2} \bullet$ Sara Machado Miranda $^{3}$ \\ Tamires Barradas Cavalcante ${ }^{4}$
}

\begin{abstract}
RESUMO
Objetiva-se analisar o conhecimento científico sobre as estratégias educativas empregadas pela enfermagem para o ensino-apredizagem das pessoas com estomia intestinal. Trata-se de uma revisão integrativa realizada nas bases de dados PubMed, LILACS e CINAHL, entre 1996 a 2014. Obteve-se amostra de 11 estudos. O Brasil liderou o numero de publicações e dos artigos incluídos na revisão e todos foram publicados em periódicos de enfermagem. Estes dados revelam o envolvimento dos enfermeiros com a temática em questão. $O$ uso de tecnologias educativas foi identificado em sete estudos que desenvolveram atividades de cunho individual e/ou em grupo por meio de uma abordagem interativa. Foi realizada em consultas ambulatoriais ou apenas pela distribuição de materiais educativos, como: cartilhas, materiais educativos impressos, cursos on-line e programas multimídias, intensificando os cuidados com pele periestoma e/ou reforçando os cuidados gerais. Para que o processo de ensino aprendizagem seja efetivo no campo da saúde, os recursos didáticos empregados devem capacitar e motivar os pacientes com vistas a conseguirem incorporar novos significados, propiciando mais autonomia. Esta revisão permitiu identificar que estratégias de educação com o uso de tecnologias educativas são mais consistentes nas mensagens transmitidas ocasionando rápida mudança de comportamento.
\end{abstract}

Palavras-chave: Estomia; Educação em Saúde; Materiais de Ensino.

\section{ABSTRACT}

The study aimed to analyze the scientific knowledge of the educational strategies used by nurses for teachinglearning programs for ostomy people. This is an integrative review carried out in the databases: PubMed, LILACS, SciELO and CINAHL from 1996 to 2014, was obtained sample of 11 studies. Brazil led the the number of publications and articles included in the review (11-100\%) were published in magazines in general nursing journals. These data reveal the nurses' involvement with the questioned theme. It was carried out in outpatient clinics or only through the distribution of educational materials, such as: booklets, printed educational materials, online courses and multimedia programs, intensifying peristaltic skin care and / or reinforcing general care. In order for the teaching-learning process to be effective in the health field, the didactic resources employed must enable and motivate patients in order to incorporate new meanings, providing more autonomy. This review identified that education strategies with the use of educational technologies are more consistent in the messages transmitted causing rapid change of behavior.

Keywords: Stoma; Health education; Teaching Materials.

\section{NOTA}

${ }^{1}$ Enfermeira graduada na Faculdade Integral Diferencial (Facid Devry); Enfermeira especialista em Estomaterapia pela Universidade Estadual do Ceará (UECE); Enfermeira assistencial e vice- presidente da Comissão de Estomaterapia do Hospital da Unimed Primavera de Teresina.Email: drika_ras@hotmail.com.

${ }^{2}$ Enfermeira especialista em Estomaterapia pela Universidade Estadual do Ceará (UECE); Enfermeira Estomaterapeuta Titulada pela Associação Brasileira de Estomaterapia: Estomias, Feridas e Incontinências (TISOBEST); Presidente da Associação Brasileira de Estomaterapia: Estomias, Feridas e Incontinências-Seção Ceará (SOBEST-CE); Membro da World Council of Enterostomal Therapists (WCET) Sociedade Internacional de Estomaterapia; Especialista em Enfermagem Médico-Cirúrgico pela UECE;Mestre em Cuidados Clínicos em Enfermagem e Saúde-PPCCLIS/UECE; Doutoranda em Cuidados Clínicos em Enfermagem e Saúde-PPCCLIS/UECE. Membro do Grupo de Pesquisa GRUPEESS-UECE Bolsista da Coordenação de Aperfeiçoamento de Pessoal de Nível Superior (Capes). Email: lucianacatundagomes@yahoo.

${ }^{3}$ Enfermeira mestre pela Universidade Federal do Piauí (UFPI); Doutoranda em Saúde Coletiva pela Universidade Federal do Maranhão (UFMA); Enfermeira do Hospital Universitario da Universidade Federal do Maranhão (HU-UFMA); Enfermeira da Maternidade Dona Evangelina Rosa da Secretaria de Saúde do Piauí - SESAPI; Email: sarammiranda2@gmail.com.

${ }^{4}$ Enfermeira mestre pela Universidade Federal do Piauí (UFPI); Enfermeira do Hospital Universitario da Universidade Federal do Maranhão (HU-UFMA); Enfermeira do Hospital do Promorar da Fundação Hospitalar de Teresina (FHT); Email: tamiresbarradas@gmail.com.

Autor responsável: Adriana Rodrigues Alves de Sousa. Estratégias educativas utilizadas para pessoas com estomia intestinal: revisão integrativa. Monografia de especialização em Estomaterapia. Teresina, Universidade Estadual do Ceará (UECE), 2015. 


\section{INTRODUÇÃO}

Estomia, também denominada estoma ou ostoma, é um termo originário do vocabulário grego para exprimir a idéia de "boca" ou abertura do segmento intestinal na parede abdominal, com o objetivo de desviar o conteúdo fecal para o meio externo ao corpo, e sua técnica consiste na abertura de um orifício que resulta do ato cirúrgico, cuja multilação é pouco visível, para evitar a passagem das fezes pela região doente ou lesada do intestino, e no qual esse orifício aberto denomina-se colostomia ou ileostomia conforme sua localização nesse órgão (intestino grosso e intestino delgado, respectivamente ${ }^{1}$.

Dentre as razões que levam à necessidade de confecção de um estoma predominam as neoplasias e os ferimentos que decorrem de arma de fogo ou branca. Esses últimos podem ser temporários, com fechamento programado para um curto período de tempo, sendo variável este tempo devido as condições relacionadas ao indivíduo que porta a bolsa, ou permanentes sujeitando ao individuo a portá-la por toda a sua vida, mediante a impossibilidade do restabelecimento do trânsito intestinal, em geral na situação de uma neoplasia maligna (câncer)².

$\mathrm{Na}$ atualidade a cirurgia de estomia constitui uma das mais importantes técnicas cirúrgicas por contribuir para a sobrevida de pessoas submetidas a confecção de estomia intestinal. Contudo, apesar das melhorias alcançadas, a nova condição de estomizado produz uma gama de alterações no estilo de vida do usuário. Independentemente de ser temporária ou definitiva, a presença de uma estomia intestinal pode resultar não só em modificações em nível fisiológico (perda do controle fecal e da eliminação de gases, complicações com a estomia e realização do autocuidado com o estoma e com a troca de bolsas coletoras), mas também em nível psicológico, emocional, sexual e social, onde cada sujeito convicto da sua cultura e história manifestará alterações que lhe são próprias ${ }^{3-4}$.

Sabe-se que a estomia intestinal traz dificuldades para quem a possui, por meio do surgimento de dúvidas e medos que iniciam desde o momento da cirurgia até a alta para o domicílio, onde a pessoa com estomia intestinal se encontra com incertezas, acreditando que suas fragilidades o impossibilitam de alcançar uma nova forma de viver e ser de maneira saudável ${ }^{5}$. Nesse contexto, torna-se fundamental a capacidade que a pessoa tem de se compreender e compreender o contexto no qual está inserida, para agir sobre si mesma e sobre sua realidade, ou seja, a construção da autonomia passa a ser imprescindível para as pessoas com estomia intestinal ${ }^{6}$.

Assim, o enfrentamento dessa nova condição de vida permeado por complexas modificações biológicas, psicossociais e econômicas requer significativos ajustes na vida do estomizado, nesse processo faz-se necessário o apoio no núcleo familiar e social, bem como na estrutura de atendimento profissional de saúde, a qual se faz essencial para a eficácia e rapidez da reabilitação do estomizado, tendo em vista a contribuição para a adaptação deste a sua nova condição $0^{3-7-8}$.

Quando se fala em coconstrução da autonomia entendese que os profissionais de saúde podem contribuir para as pessoas ampliarem seus conhecimentos atuando com o propósito de explicitar a dimensão técnica envolvida no seu processo de saúde. Assim o profissional enfermeiro tem papel importante no auxílio das pessoas com estomia intestinal, objetivando ampliarem seu conhecimento, incentivando sua capacidade reflexiva, interferindo em sua rede de dependência e na sua tomada de decisões?.

0 cuidado de enfermagem à pessoa com estomia intestinal compreende desde o momento da avaliação diagnóstica, onde é definido a necessidade da confecção do estoma, no pré-operatório, no trans-operatório, no pós-operatório imediato e no tardio ou fase ambulatorial, na qual se evidencia a qualidade de vida, autonomia e reabilitação. Dessarte, para o enfermeiro desenvolver um cuidado autêntico e de qualidade, é imperioso eleger em suas ações aspectos substanciais à relação humano-humano como: a conversa, a escuta, o toque, a demonstração de preocupação e afeto ${ }^{10}$.

Torna-se mister reconhecer que a educação em saúde é um instrumento fundamental para uma assistência de enfermagem de boa qualidade, onde o enfermeiro, como educador em saúde, necessita orientar os pacientes e seus familiares. Desse modo, entendemos a educação em saúde como um processo de ensino que o enfermeiro faz com seus clientes, objetivando o aprendizado e o autocuidado ${ }^{11}$.

Em educação e saúde, o fundamental é a escolha por uma concepção pedagógica capaz de desenvolver no paciente a possibilidade crítica e a construção de novos conhecimentos. No entanto, segundo se verifica no cotidiano das práticas de saúde desenvolvidas junto às pessoas com estomias intestinais, podemos encontrar várias formas de tecnologias que promovem a emancipação dos sujeitos envolvidos no cuidar. Dentre elas, a tecnologia educativa surge como instrumento facilitador do processo ensino-aprendizagem, ocasionando o desenvolvimento de habilidades, sendo mediadora de conhecimentos para o cuidado ${ }^{12}$.

A inserção das tecnologias no contexto da educação em saúde complementa as ações desempenhadas pelo profissional enfermeiro na sua relação com a pessoa com estomia intestinal, assim o uso de tecnologias que contribuam com a educação em saúde abre novos caminhos no processo de ensino-aprendizagem por meio de interações mediadas pelo locutor (enfermeiro), leitor (estomizado e familiares) e o objeto do discurso (material educativo) ${ }^{12}$.

$\mathrm{Na}$ condição de educador em saúde, o profissional enfermeiro deve implementar cuidados à pessoa com estomia intestinal, por meio de uma interação mútua, horizontal e humanizada, transportando essa pessoa da condição de mero receptor de informações para autor (sujeito transformador) de sua autonomia. Diante disso, dá-se a importância da educação em saúde, por meio da 
informação compartilhada pela ação educativa, através da prática dialógica e do uso de tecnologias educativas, com vistas permitir que o processo de ensino-aprendizagem se concretize e o cliente exerça sua função de sujeito, independente e autônomo.

A presença da relevância do uso, pela enfermagem, de tecnologias nas atividades educativas, efetuou-se uma revisão integrativa com o objetivo de analisar o conhecimento científico produzido sobre as estratégias de educação em saúde utilizadas para o ensino-aprendizagem das pessoas com estomia intestinal.

\section{MÉTODO}

Trata-se de uma revisão integrativa de literatura que visa à busca de pesquisas já realizadas e consiste na síntese de múltiplos estudos que permitem conclusões gerais a respeito de uma área ${ }^{13}$. A partir do momento que possibilita a síntese de conhecimentos dos estudos incluídos na revisão, facilita aos profissionais de saúde que prestam cuidados, a tomada de decisões nas intervenções para um cuidado mais efetivo consolidando a prática clínica ao embasamento científico ${ }^{14}$. 0 estudo fundamenta-se em princípios da revisão integrativa proposta por Ganong $(1987)^{15}$.

Nesse sentido, a pergunta norteadora para este estudo foi: quais as estratégias de educação em saúde utilizadas para o ensino-aprendizagem de pessoas com estomia intestinal? Nessa perspectiva, foi realizada busca na literatura científica durante o mês de outubro de 2015. 0 acesso deu-se nas bases de dados: National Library of Medicine (PubMed/Medline), Literatura Latino-Americana e do Caribe em Ciências da Saúde (LILACS) e Cumulative Index of Nursingand Allied Health Literature (CINAHL). Empregou-se a terminologia em saúde consultada nos Descritores em Ciências da Saúde (DECS)/BIREME e do
Medical Subject Headings (MeSH)/PubMed, utilizando os seguintes descritores cruzados com o marcador boleano "and": estomia/ostomy, educação em saúde/health education, materiais de ensino/teaching materials.

No momento da busca, cada base de dados foi acessada em um único dia, sendo pesquisada em um único momento de modo a esgotar as buscas das publicações e evitar viés que prejudicasse a fidedignidade dessa etapa. Ademais, a referida etapa foi realizada por dois pesquisadores, de forma independente, com posterior reunião para consenso sobre os artigos que iriam compor a amostra. Foram incluídos os artigos que abordavam temas sobre estomia intestinal, educação em saúde e materiais de ensino, publicado em português, inglês e espanhol, disponíveis na íntegra, que responderam a questão norteadora dessa revisão e com recorte temporal de 1996 a 2014, em razão de poucos artigos tratarem sobre este tema houve a necessidade de manter recorte temporal maior.

Foram excluídas publicações do tipo editorial, dissertações, revisões de literatura, estudos que não abordavam a temática e publicações duplicadas, das quais foi selecionado o artigo apenas uma vez. Durante a busca identificamos 82 artigos no LILACS, 83 artigos na CINAHL, não sendo encontrado nenhum artigo no PubMed, totalizando 166 artigos. Restaram 11 artigos, os quais após leitura minuciosa formaram a amostra para o estudo. No fluxograma 1 são apresentados os números de artigos encontrados, excluídos e selecionados por bases de dados.

Para definir as informações a serem extraídas dos estudos, efetuou-se uma leitura minuciosa e analítica com auxílio do formulário adaptado e registrou-se os seguintes aspectos: identificação do estudo (título do artigo, título do periódico, autores, países, idioma, ano de publicação e nível de evidência científica); tipo de revista; características metodológicas do estudo (tecnologia utilizada/desenvolvida, público-alvo) ${ }^{16}$. A determinação

Fluxograma 1. Distribuição do número de artigos encontrados, excluídos e selecionados por bases de dados. Teresina-PI, 2015

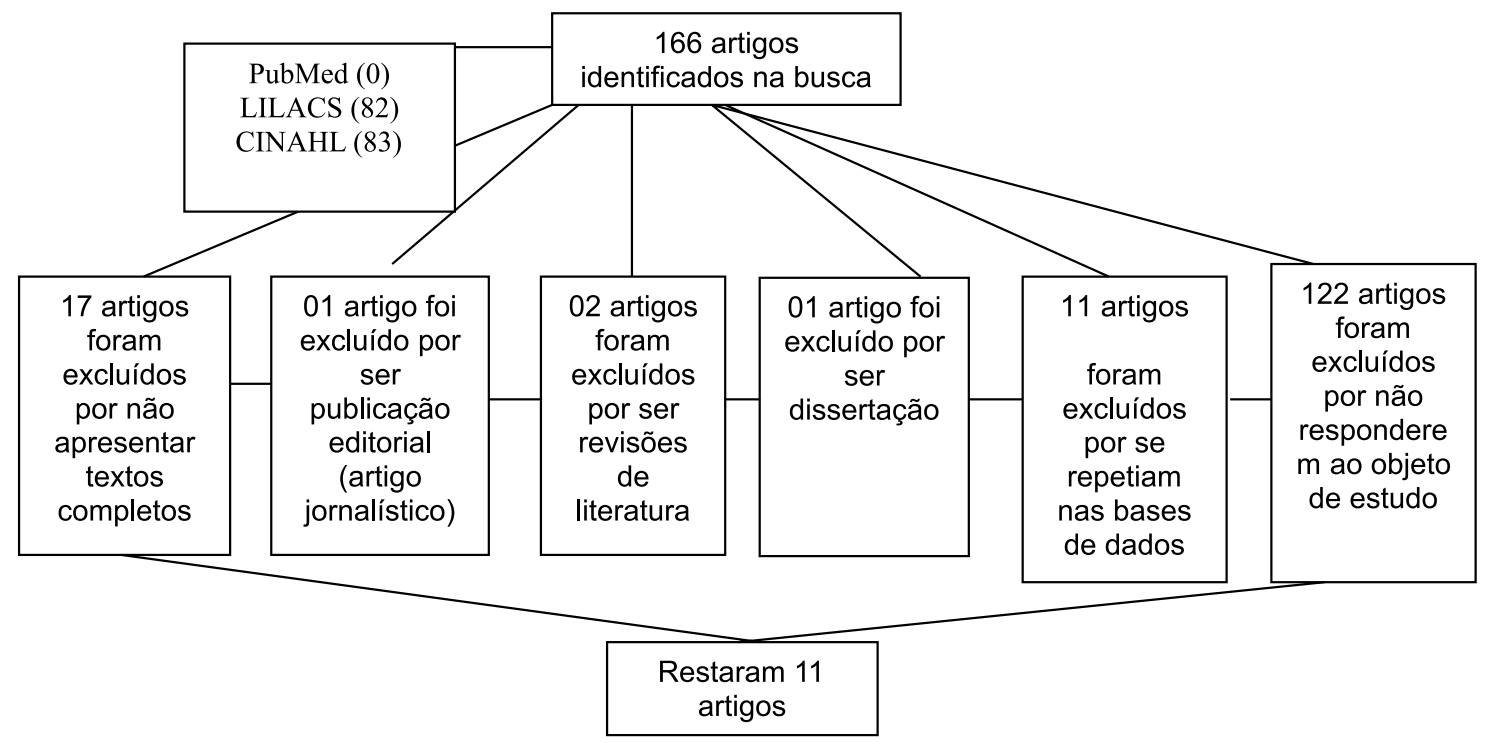

Fonte: elaborado pela autora. 
do nível de evidência dos estudos foi realizada com base na classificação revista das forças de evidência, proposta por Polit e Beck ${ }^{17}$, que considera as evidências em seis níveis, sendo: nível I, estudos relacionados com a metanálise de múltiplos estudos controlados; nível II estudos experimentais individuais; nível III, estudos quaseexperimentais, como ensaio clínico não randomizado, grupo único pré e pós teste, além de séries temporais ou caso-controle; nível IV, estudos não experimentais, como pesquisa descritiva, correlacional e comparativa, com abordagem qualitativa e estudos de caso; nível V, dados de avaliação de programas e obtidos de forma sistemática e nível VI, opiniões de especialistas, relatos de experiência, consensos, regulamentos e legislações ${ }^{17}$. Pela sua natureza, o presente estudo não envolveu seres humanos. Consideramos o respeito pela propriedade intelectual dos autores dos artigos que constituíram a amostra, nomeadamente, na citação rigorosa dos seus trabalhos.

\section{RESULTADOS}

No quadro 1, são apresentados os resultados das publicações quanto as características, à autoria do estudo, ano de publicação, país de origem, título, periódico, delineamento de pesquisa, nível de evidência e as estratégias de educação em saúde realizadas.

Pela análise dos artigos selecionados, a grande parte dos estudos foi encontrada na base de dados LILACS (10-91\%), com maior concentração e maior frequência nos últimos cinco anos, uma vez que a partir de 2010 encontramos mais artigos e com menor intervalo de tempo de publicação entre um e outro, com predomínio

Quadro 1. Caracterização das pesquisas e estratégias de educação em saúde realizadas. Teresina-PI, Brasil, 2015

\begin{tabular}{|c|c|c|c|c|c|}
\hline Número/Autor & Ano/País & Periódico & $\begin{array}{c}\text { Delineamento da } \\
\text { pesquisa* }\end{array}$ & $\begin{array}{c}\text { Nível de } \\
\text { Evidência** }\end{array}$ & Estratégias de Educação em Saúde \\
\hline 1. Coey, L. & 1996 Inglaterra & $\begin{array}{l}\text { Journal of } \\
\text { Clinical Nursing }\end{array}$ & $\begin{array}{c}\text { Tipo de estudo } \\
\text { não identificado }\end{array}$ & - & $\begin{array}{l}\text { Ação educativa através de materiais } \\
\text { impressos (PEM) }\end{array}$ \\
\hline 2. O'Shea, H. S. & $\begin{array}{l}2001 \text { Estados } \\
\text { Unidos }\end{array}$ & J.WOCN & $\begin{array}{l}\text { Tipo de estudo } \\
\text { não identificado }\end{array}$ & - & $\begin{array}{l}\text { Ação prática de enfermagem } \\
\text { baseada no processo de } \\
\text { aprendizagem }\end{array}$ \\
\hline 3. Lo, S. F. et al. & $2010^{a}$ China & $\begin{array}{c}\text { Journal of } \\
\text { Clinical Nursing }\end{array}$ & $\begin{array}{l}\text { Estudo } \\
\text { Randomizado }\end{array}$ & II & $\begin{array}{l}\text { Ação educativa através de programa } \\
\text { de multimídia de aprendizagem }\end{array}$ \\
\hline 4. Slater, R. C. & 2010 Inglaterra & $\begin{array}{l}\text { British Journal } \\
\text { of Community } \\
\text { Nursing }\end{array}$ & $\begin{array}{l}\text { Tipo de estudo } \\
\text { não identificado }\end{array}$ & - & $\begin{array}{l}\text { Ação educativa através de } \\
\text { orientações verbais e escritas e } \\
\text { escala (The Stoma Quality of Life } \\
\text { Scale) }\end{array}$ \\
\hline 5. Lo, S. F. et al. & 2010b China & $\begin{array}{l}\text { Journal of } \\
\text { Advanced } \\
\text { Nursing }\end{array}$ & $\begin{array}{l}\text { Estudo } \\
\text { randomizado } \\
\text { experimental }\end{array}$ & II & $\begin{array}{l}\text { Ação educativa através do uso de } \\
\text { um programa multimídia de ensino }\end{array}$ \\
\hline $\begin{array}{l}\text { 6. Martins, P. A. F.; } \\
\text { Alvim, N. A. T. }\end{array}$ & 2011 Brasil & Rev. Bras. Enferm. & $\begin{array}{l}\text { Tipo } \\
\text { convergente- } \\
\text { assistencial }\end{array}$ & IV & $\begin{array}{l}\text { Ação educativa através da prática } \\
\text { dialógica do enfermeiro }\end{array}$ \\
\hline $\begin{array}{l}\text { 7. Gemmill, R. } \\
\text { et al. }\end{array}$ & $\begin{array}{l}2011 \text { Estados } \\
\text { Unidos }\end{array}$ & $\begin{array}{l}\text { Journal of } \\
\text { Continuing } \\
\text { Education in } \\
\text { Nursing }\end{array}$ & Estudo descritivo & IV & $\begin{array}{l}\text { Ação educativa através de folheto } \\
\text { informativo, vídeo instrutivo, } \\
\text { demonstração de cuidados e } \\
\text { acompanhamento no domicílio }\end{array}$ \\
\hline $\begin{array}{l}\text { 8. Martins, P. A. F.; } \\
\text { Alvim, N. A. T. }\end{array}$ & 2012 Brasil & $\begin{array}{l}\text { Texto Contexto } \\
\text { Enferm. }\end{array}$ & $\begin{array}{l}\text { Qualitativo tipo } \\
\text { convergente- } \\
\text { assistencial }\end{array}$ & IV & $\begin{array}{l}\text { Plano de cuidados compartilhado } \\
\text { mediado pela prática dialógica }\end{array}$ \\
\hline $\begin{array}{l}\text { 9. Barros, E. J. L. } \\
\text { et al. }\end{array}$ & 2012 Brasil & $\begin{array}{l}\text { Rev. Gaucha } \\
\text { Enferm }\end{array}$ & Descritivo & IV & $\begin{array}{l}\text { Dialogo mediado por uma } \\
\text { tecnologia educativa (cartilha } \\
\text { educativa) }\end{array}$ \\
\hline $\begin{array}{l}\text { 10. Poletto, D, } \\
\text { Silva, D. M. G. V. }\end{array}$ & 2013 Brasil & $\begin{array}{l}\text { Rev. Latino Am. } \\
\text { Enferma-gem }\end{array}$ & Descritivo & IV & $\begin{array}{l}\text { Informações, orientações e } \\
\text { demonstrações técnicas de forma } \\
\text { verticalizada pela equipe de saúde, } \\
\text { especial-mente da enfermagem }\end{array}$ \\
\hline $\begin{array}{l}\text { 11. Souza, N. V. D. } \\
\text { O. et al. }\end{array}$ & 2014 Brasil & J. Bras. Tele & $\begin{array}{l}\text { Relato de } \\
\text { experiência }\end{array}$ & VI & $\begin{array}{l}\text { Tecnologias de comunicação e } \\
\text { informações virtuais por meio de } \\
\text { palestras e cursos on-line }\end{array}$ \\
\hline
\end{tabular}

Fonte: Elaborado pela autora.

${ }^{*}$ Os delineamentos enumerados neste quadro estão descritos como no artigo analisado.

${ }^{*}$ O nível de evidência dos estudos foi determinado segundo a classificação de Polit e Beck ${ }^{(17)}$. 
no ano de 2010 e 2012 (representando respectivamente 30\% em 2010 e 20\% em 2012). O Brasil liderou o número de publicações (5-45,5\%). Dois estudos foram produzidos na América do Norte (2-18,2\%), cinco estudos produzidos na América do Sul (5-45,5\%), dois estudos na Europa (2-18,2\%) e dois estudos na Ásia (2-18,2\%) a evidenciar a distribuição das pesquisas em todo mundo. Dos artigos incluídos na revisão, 11 (100\%) foram publicados em periódicos de enfermagem em geral.

Quanto ao delineamento da pesquisa, evidenciou-se: estudo randomizado, descritivo, convergente assistencial e relato de experiência. Três artigos analisados não foram possíveis identificar o tipo de estudo. Em relação às forças de evidências observou-se que $45,45 \%$ das publicações possuem o nível de evidência IV, seguidas de $18 \%$ com nível de evidência II e $9 \%$ com nível VI. Não foi encontrado nas publicações, trabalhos com níveis de evidência I, II e V. Em relação aos tipos de estratégias educativas que têm sido utilizadas para a abordagem das pessoas com estomia intestinal, foi possível observar a existência de duas mais expressivas: a prática dialógica e a orientação por meio de tecnologias educativas.

\section{DISCUSSÃO}

Para melhor discutir o enfoque educativo dos estudos analisados, optou-se pela organização dos artigos agrupados em duas categorias: a categoria Educação em saúde por meio da prática dialógica (04 estudos) e Educação em saúde com uso de tecnologias educativas (07 estudos).

\section{Educação em saúde por meio da prática dialógica}

Nesta categoria foram agrupados quatro estudos que abrangeram questões referentes às atividades de educação em saúde por meio da prática dialógica que se faz imperiosa para a educação em saúde da pessoa com estomia intestinal, uma vez que o diálogo é a essência da educação. De acordo com Freire ${ }^{18}$, o homem é o sujeito de sua própria educação. Assim sendo, o homem como sujeito cognoscente inserido no mundo e com o mundo deve ser reconhecido e ouvido através de um processo de educação mediado por uma relação horizontal e recíproca, instalando-se um processo participativo, dialogal e ativo, que permite comunicação e educação em comunhão ${ }^{19}$.

Nesse contexto, é por meio da dialogicidade que os sujeitos alcançam a comunicação. O diálogo é mediatizado por uma interação horizontal e mútua, permitindo o compartilhamento de saberes e práticas entre os sujeitos envolvidos através das palavras, levando em consideração o saber do outro, reconhecendo suas crenças, cultura, contexto social, político e econômico, dessa forma se compreende que a educação não ocorre de forma depositária, unidirecional e através de discurso monológico ${ }^{19}$.
Desta maneira, a educação em saúde é um processo permanente com princípios críticos e reflexivos e metodologia baseada no dialogo construindo indivíduos ativos e participativos nas questões de gestão da saúde ${ }^{20}$. Porém de acordo com Poletto e Silva ${ }^{21}$, em estudo desenvolvido com pessoas com estomia intestinal e familiares cuidadores, foi predominante o processo educativo com base na tendência tradicional, com orientações e informações ditadas. Havendo somente uma transferência de informações, pois o aprendizado só acontece mediado por troca de conhecimentos.

Por meio deste estudo eles incentivam os profissionais a substituírem o trabalho técnico e o atendimento verticalizado pela prática do diálogo, da criação de vínculo e da educação em saúde ${ }^{21}$. A relação de diálogo para uma troca de saberes permite o surgimento de um terceiro saber, resultado da interação entre os conhecimentos dos profissionais de saúde e da população, uma vez que o saber popular é um meio para ampliar as práticas dos profissionais de saúde promovendo vivências e experiências das pessoas com estomia intestinal, contribuindo para a construção de um plano de cuidado individual e congruente com as necessidades e desejo do estomizado ${ }^{22}$. Segundo O'Shea ${ }^{23}$, a chave para o sucesso do processo de ensino-aprendizagem é a aceitação da legitimidade e crenças do outro, o respeito pela autonomia de cada um e a preocupação genuína com o bem-estar da pessoa com estomia intestinal. Assim o diálogo através da ação profissional possibilita o alcance da autonomia, a ruptura da cultura do silêncio e a libertação da pessoa com estomia intestinal antes vista como objeto ${ }^{24}$.

A educação em saúde embasada no ensino dialógico a essas pessoas equivale uma ferramenta indispensável para o desenvolvimento de capacidades individuais e coletivas visando à melhoria da qualidade de vida e saúde ${ }^{25}$. As práticas educativas em saúde no contexto da enfermagem vêm sendo uma realidade cada vez mais efetivada devido à mudança de paradigmas de atenção à saúde, desse modo a Organização Mundial de Saúde ${ }^{26}$, sugere como práticas educativas em saúde a realização de workshops educacionais e de capacitação para pacientes e familiares, a utilização de materiais educativos para a complementação das orientações aos pacientes e às famílias com acesso à informação e apoio ao autogerenciamento fora do estabelecimento de saúde utilizando telefone ou Internet, assim como, o uso de recursos computadorizados para a preparação de materiais individualizados de autogerenciamento.

Diante disso, a educação em saúde e a inserção de outras tecnologias educacionais nas práticas educativas à pessoa com estomia intestinal vêm para complementar as ações desempenhadas pelo enfermeiro bem como abrir novas alternativas no processo ensino-aprendizagem ${ }^{27}$. De tal modo, o diálogo mediado por uma tecnologia educativa equivale à forma de cuidado humanizado fomentador da emancipação dos sujeitos ${ }^{5}$. 


\section{Educação em saúde com o uso de tecnologias educativas}

Essa estratégia educativa foi identificada em sete estudos que desenvolveram atividades de cunho individual e/ou em grupo por meio de uma abordagem interativa. Foi realizada em consultas ambulatoriais ou apenas pela distribuição de materiais educativos, como: cartilhas, materiais educativos impressos, cursos on-line e programas multimídias, intensificando os cuidados com pele periestoma e/ou reforçando os cuidados gerais.

A tecnologia educacional compreende o resultado de processos concretizados a partir da experiência cotidiana e da pesquisa, para a formação de um conjunto de conhecimentos científicos para a construção de produtos materiais, ou não, com o propósito de provocar intervenções sobre uma determinada situação prática. Todo esse processo deve ser avaliado e controlado sistematicamente ${ }^{28}$. Portanto, na prática do cuidado, a enfermagem possui possibilidades de encontrar formas de tecnologias que provoquem o processo de emancipação dos sujeitos envolvidos no cuidar. Assim, as tecnologias vinculadas à educação se identificam com métodos de cuidados simplificados com o objetivo de tornar sua prática comum, facilitando o autocuidado ${ }^{29}$.

Entendemos que o enfermeiro como educador deve desenvolver práticas que facilitem a aprendizagem significativa, onde o uso de tecnologias educativas são estratégias importantes para a execução das atividades em saúde além de promover de forma mais ampla e criativa o seu real papel de orientador e facilitador ${ }^{30}$. Assim as tecnologias educacionais, utilizadas pela enfermagem, são compreendidas com um fundamento filosófico voltado para o desenvolvimento do indivíduo e caracterizada por novas teorias, ensinos, pesquisas, conceitos, técnicas para a atualização da educação, possibilitando ao educador maneiras inovadoras por meio de imagens, permutando conhecimentos, facilitando o aprendizado e contribuindo para o avanço educacional ${ }^{31}$.

No estudo de $\mathrm{Coey}^{32}$ que relata sobre a legibilidade de materiais educativos impressos utilizados para informar as pessoas portadoras de estomias, evidencia que materiais educativos impressos são muito eficientes na educação dos pacientes, uma vez que, são reutilizáveis, permanentes, legíveis no ritmo do leitor, fáceis de reproduzir e distribuir, consistentes em mensagem transmitida e portáteis, então ao receberem informações escritas, têm a ansiedade reduzida e vão para casa mais cedo ${ }^{32}$.

Conforme o estudo de Barros et $a l^{5}$ a aplicação de um programa de educação em saúde individual por meio de cartilha educativa realizado numa instituição pública com pessoas com estomia intestinal após intervenção educativa, mostrou mudança de atitude quanto ao conhecimento sobre os cuidados com a pele periestoma ${ }^{5}$. Partindo dessa premissa, a tecnologia educacional tem o intuito de educar, no entanto, somente será considerada educacional se alcançar este objetivo, então o enfermeiro assume importante papel perante às diversas tecnologias educativas que podem ser apresentadas, uma vez que as mesmas devem estar em consonância com o público ao qual se dirige levando em consideração o nível social, cultural, escolaridade preocupando-se com o conteúdo a ser expresso, como será mostrado e como aquele novo aprendizado poderá repercutir na vida da pessoa com estomia intestinal ${ }^{33}$.

Dentre essas diferentes estratégias e cientes da existência de várias tecnologias do cuidado na saúde, não podemos deixar de abordar a utilização de imagens por meio de multimídia e cursos on-line, responsável pela produção, muito rápida, de mudanças de comportamento ${ }^{34}$. $O$ estudo de Lo et a $l^{35}$ menciona a avaliação da eficácia do Programa de Educação Multimídea (MEP) para pacientes com estoma, relatando claramente que a intervenção melhorou significativamente os níveis de conhecimento dos pacientes com estoma, construindo um conhecimento para possíveis mudanças de comportamento, onde demonstraram que a intervenção educacional empregando uma abordagem multimídea está ligada ao aumento e melhores atitudes de autocuidado ${ }^{35}$. Ainda nesse mesmo estudo, autores afirmam que o programa de aprendizagem multimídia educação (MLEP) é mais eficaz e com menor custo que um programa de serviço de educação convencional (CESP) ${ }^{36}$.

De acordo com Souza et $a^{37}$, a ampliação e consolidação do conhecimento por meio de um curso on-line abordando a problemática biopsicossocial das pessoas estomizadas contribuiu para o acesso técnico e científico de indivíduos residentes em diversas áreas do país, além da ampliação de conhecimentos trazendo os avanços da estomaterapia e melhorando $o$ atendimento das pessoas. Nesse contexto, as multimídias podem constituir-se em formas de dinamizar o autocuidado das pessoas estomizadas. Dessa maneira, para Lo et $a^{35}$, materiais educacionais como vídeos $\mathrm{e}$ imagens permitem a exposição sistemática e repetitiva dos detalhes de determinada técnica ou assunto, favorecendo sua compreensão e memorização.

\section{CONCLUSÃO}

As estratégias de educação em saúde para pessoas com estomia intestinal têm sido foco crescente nos últimos anos. Foi possível observar o predomínio de estratégias por meio de tecnologias educativas. Estratégias que envolvem recursos tecnológicos modernos nem sempre se tornam viáveis em consequência do nível educacional do paciente e a dificuldade de manipular tais materiais. De forma que ainda é de grande importância o ensino baseado numa prática dialógica, bem como a disponibilidade dos recursos comunitários e das instituições de saúde através de atendimento especializado pelos profissionais.

Nessas perspectivas, é mister ultrapassar as estratégias educativas em saúde focadas apenas na transmissão de 
informação, e apropriar-se de uma abordagem críticosocial, que busque estimular a participação das pessoas com estomia intestinal de maneira ativa em todas as fases do processo educativo, desde seu planejamento até sua avaliação. Dessa maneira, o estudo sobre as estratégias educativas utilizadas para as pessoas com estomia intestinal mostrou que as tecnologias se fazem presentes seja através da prática dialógica, das tecnologias educativas ou de ambas associadas. Porém, na prática assistencial do enfermeiro, as lacunas na assistência a pessoa com estomia intestinal permanecem em todos os âmbitos de atuação do enfermeiro.

Entende-se a necessidade de mais estudos que explicitem o uso de tecnologias educativas na prática do enfermeiro, comprovando os benefícios que trazem às pessoas portadoras de estomias intestinais, bem como estudos que validem as mesmas, para que assim o uso das mesmas superem a transmissão de informações na assistência de enfermagem à pessoa com estomia intestinal.

\section{REFERÊNCIAS}

1. Associação Brasileira de Ostomizados. Quantitativo aproximado de pessoas ostomizadas no Brasil. Rio de Janeiro [Internet] 2014. [acesso em 2 abr. 2014]. Disponível em: <http://www.abraso.org.br/estatistica_ostomizados.htm>.

2. Belato R, Maruyama SAT, Silva CM, Castro PA. Condição crônica ostomia e as repercussões que traz para a vida da pessoa e sua família. Cien. Cuid. Saúde. 2007; 6(1):40-50.

3. Cascais AFMV, Martini JG, Almeida PJS. O impacto da ostomia no processo de viver humano. Texto Contexto Enferm. 2007;16(1):163-167.

4. Dázio EMR. O significado do estoma intestinal entre homens: um estudo etnográfico [Tese]. Escola de Enfermagem de Ribeirão Preto, Universidade de São Paulo, Ribeirão Preto; 2008.

5. Barros EJL, Santos SSC, Gomes GC, Erdmann AL. Gerontotecnologia educativa voltada ao idoso estomizado à luz da complexidade. Rev. Gaúcha Enferm. 2012;33(2):95-101.

6. Campos GWS. Clínica e saúde coletiva compartilhadas: teoria Paidéia e reformulação ampliada do trabalho em saúde. In: Campos GWS, Minayo MCS, Akerman M, Drumond MJ, Carvalho YM. (Orgs.). Tratado de saúde coletiva. São Paulo: Hucitec; Rio de Janeiro: Fiocruz; 2006. p. 41-81.

7. Santos VLCG, Cesaretti IUR. Assistência em estomaterapia: cuidando de pessoas com estomias. São Paulo: Atheneu, 2015.

8. Sonobe HM, Barichello E, Zago MMF. A visão do colostomizado sobre o uso da bolsa de colostomia. Revista Brasileira de Cancerologia. 2002;8(3):341-348.

9. Onocko CRT, Campos GWS. Co-construção de autonomia: o sujeito em questão. In: Campos GWS, Minayo MCS, Akerman M, Drumond MJ, Carvalho YM. (Orgs.) Tratado de saúde coletiva. São Paulo: Hucitec; Rio de Janeiro: Fiocruz; 2006. p. 669-88.

10. Silva DC, Alvim NAT, Figueiredo PA. Tecnologias leves em saúde e sua relação com o cuidado de enfermagem hospitalar. Esc. Anna Nery Rev. Enferm. 2008;12(2):291-198.
11. Reveles AG, Takahashi RT. Educação em saúde ao estomizado: um estudo bibliométrico. Rev. Esc. Enferm. USP. 2007;41(2): 245-250.

12. Souza AC, Colomé ICS, Costa LED, Oliveira DLLC. A educação em saúde com grupos na comunidade: uma estratégia facilitadora da promoção da saúde. Rev. Gaúcha Enferm. 2005;26(2):147-153.

13. Mendes KDS, Silveira RCCP, Galvão CM. Revisão integrativa: método de pesquisa para a incorporação de evidências na saúde e na enfermagem. Texto Contexto Enferm. 2008;17(4):758-764.

14. Souza M T, Silva MD, Carvalho R. Revisão integrativa: o que é e como fazer. Einstein. 2010;8(1):102-106.

15. Ganong LH. Integrative reviews of nursing research. Res. Nurs. Health. 1987;10(1):1-11.

16. Ursi ES. Prevenção de lesões de pele no perioperatório: revisão integrativa da literatura [dissertação]. Escola de Enfermagem de Ribeirão Preto, Universidade de São Paulo, Ribeirão Preto; 2005.

17. Polit DF; Beck CT. Fundamentos de pesquisa em enfermagem: avaliação de evidências para a prática da Enfermagem. 7. ed. Porto Alegre: Artmed; 2011.

18. Freire. Educação e mudança. 20.ed. São Paulo: Paz e Terra; 1994. p $54-60$.

19. Martins PAF. Compartilhando saberes e práticas com clientes estomizados acerca da manutenção da estomia de eliminação: uma proposta educativa do cuidado de enfermagem no contexto ambulatorial. [dissertação]. Programa de Pós-Graduação em Enfermagem, Escola de Enfermagem Anna Nery, Universidade Federal do Rio de Janeiro, Rio de Janeiro; 2010.

20. Brasil. Ministério da Saúde. Conselho Nacional das Secretarias Municipais de Saúde. O SUS de A a Z: garantindo saúde nos municípios. 3. ed. Brasília: Ministério da Saúde; 2009. p. 480.

21. Poletto D, Silva DMGV. Living with intestinal stoma: the construction of autonomy for care. Rev. Latino-Am. Enfermagem, Ribeirão Preto. 2013;21(2):531-538.

22. Gomes LB, Merhy EE. Compreendendo a educação popular em saúde: um estudo na literatura brasileira. Cad. Saúde Pública. 2011;27(1):7-18.

23. O'Shea HS. Teaching the adult ostomy patient. J Wound Ostomy Continence Nurs. 2001;28(1):47-54.

24. Martins PAF, Alvim NAT. Plano de cuidados compartilhado junto a clientes estomizados: a pedagogia freireana e suas contribuições à prática educativa. Texto Contexto Enferm. 2012;21(2):286-294.

25. Silva LD, Beck CLC, Dissen CM, Tavares JP, Budó MLD, Silva HS. O enfermeiro e a educação em saúde: um estudo bibliográfico. Rev. Enferm. UFSM. 2012;2(2):412-419.

26. Organização Mundialo da Saúde. Cuidados inovadores pra condições crônicas: componentes estruturais de ação. Relatório anual. Brasília; 2003.

27. Freitas AAS, Cabral IE. O cuidado á pessoa traqueostomizada: análise de um folheto educativo. Esc. Anna Nery Rev. Enferm. 2008;12(1):84-89.

28. Teixeira E. Tecnologias em Enfermagem: produções e tendências para a educação em saúde com a comunidade. Rev. Eletr. Enf. 2010;12(4):598. 
29. Merhy EE. Em busca de ferramentas analisadoras das In: Merhy EE, Onocko R. (Orgs.). Agir em saúde: um desafio para o público. 2. ed. São Paulo: Hucitec; 2002. p. 113-150.

30. Litwin E. Educação a distância: temas para o debate de uma nova agenda educativa. Porto Alegre: ArtMed; 2001.

31. Nietsche EA, Backes VMS, Colomé CLM, Ceratti RN, Ferraz F. Tecnologias educacionais, assistenciais e gerenciais: uma reflexão a partir da concepção dos docentes de enfermagem. Rev Latino-Am. Enfermagem. 2005;13(3):344-53.

32. Coey L. Readability of printed educational materials used to inform potential and actual ostomates. J. Clin. Nurs. 1996:5(6):359-366.

33. Áfio ACE, Balbino AC, Alves MDS, Carvalho LV, Santos MCL, Oliveira NR. Análise do conceito de tecnologia educacional em enfermagem aplicada ao paciente. Rev. Rene. 2014;15(1):158-165.

34. Moraes AF. Cultural diversity in health-related videos. Interface - Comunic., Saúde, Educ. 2008;12(27):811-22.

35. Lo SF, Wang YT, Wu LY, Hsu MY, Chang SC, Hayter M. Multimedia education programme for patients with a stoma: effectiveness evaluation. J. Adv. Nurs. 2010;67(1):68-76.

36. Lo SF, Wang YT, Wu LY, Hsu MY, Chang SC, Hayter M. A costeffectiveness analysis of a multimedia learning education program for stoma patients. J. Clin. Nurs. 2010;19(1314):1844-1854.

37. Souza NVDO, David HMSL, Mauricio VC, Costa CCP. Enfermagem em estomaterapia no Telessaúde UERJ: relato de experiência. J. Bras. Tele. 2014;3(2):70-72. 